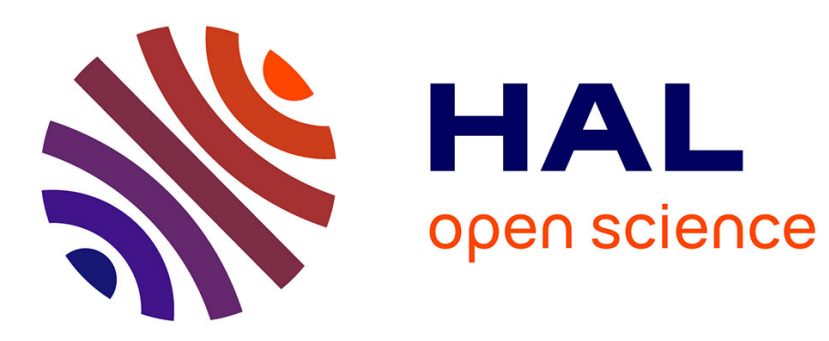

\title{
Inverse problem in electrocardography via the factorization method of boundary value problems
}

Julien Bouyssier, Nejib Zemzemi, Jacques Henry

\section{To cite this version:}

Julien Bouyssier, Nejib Zemzemi, Jacques Henry. Inverse problem in electrocardography via the factorization method of boundary value problems. IEEE 12th International Symposium on Biomedical Imaging (ISBI), 2015, Apr 2015, New York, France. 10.1109/ISBI.2015.7163979 hal-01222401

\section{HAL Id: hal-01222401 \\ https://hal.science/hal-01222401}

Submitted on 29 Oct 2015

HAL is a multi-disciplinary open access archive for the deposit and dissemination of scientific research documents, whether they are published or not. The documents may come from teaching and research institutions in France or abroad, or from public or private research centers.
L'archive ouverte pluridisciplinaire HAL, est destinée au dépôt et à la diffusion de documents scientifiques de niveau recherche, publiés ou non, émanant des établissements d'enseignement et de recherche français ou étrangers, des laboratoires publics ou privés. 


\title{
INVERSE PROBLEM IN ELECTROCARDOGRAPHY VIA THE FACTORIZATION METHOD OF BOUNDARY VALUE PROBLEMS
}

\author{
Julien Bouyssier ${ }^{\star \dagger *} \quad$ Nejib Zemzemi ${ }^{\star \dagger *} \quad$ Jacques Henry ${ }^{\star \dagger *}$ \\ * INRIA, équipe Carmen, Centre Bordeaux Sud-Ouest, France \\ †LIRYC, L'Institut de Rythmologie et Modélisation Cardiaque, Université de Bordeaux \\ *IMB, Institut de Mathématiques de Bordeaux UMR 5251
}

\begin{abstract}
We present a new mathematical approach for solving the inverse problem in electrocardiography. This approach is based on the factorization of boundary value problems method. In this paper we derive the mathematical equations and test this method on synthetical data generated on realistic heart and torso geometries using the state-of-the-art bidomain model in the heart coupled to the Laplace equation in the torso. We measure the accuracy of the inverse solution using spatial Relative Error (RE) and Correlation Coefficient (CC).
\end{abstract}

Index Terms - Inverse problem, electrocardiography, Riccati equations, factorization method, boundary value problems, electrocardiagraphic imaging (ECGI).

\section{INTRODUCTION}

ECGI is a new imaging technique that noninvasively images cardiac electrical activity on the heart surface. In ECGI, a multi-electrode vest records body-surface potential maps (BSPMs). Then, using BSPMs, geometrical information from CT-scans, a mathematical modelling and a numerical algorithm, we reconstruct electrical potentials, electrograms and activation maps on the heart surface [1, 2, 3]. In this work we develop a new method called the factorization of boundary value problems method $[4,5,6]$ based on the invariant embedding approach. The electrical potential in the ECGI inverse problem is governed by the Laplace equation with a homogeneous Neumann boundary condition and an extra Dirichlet boundary condition on the body surface. We decompose this problem into two boundary value problems and treat it as an optimal control problem. In [7], this optimal control problem is solved using a minimization procedure of a cost function, allowing to compute both electrical potential and its normal derivative over the heart surface. In our approach, the two sub-problems are embedded into a family of similar problems on subdomains bounded by a boundary moving from the torso skin to the heart surface. We can

This work is sponsored from the french government managed by the National Research Agency under the program "Investissements d'Avenir" by the grant number ANR-10-IAHU-04. define Neumman-Dirichlet and Dirichlet-Neumann operators on this moving boundary that satisfy Riccati equations and which need to be solved from the torso surface to the heart surface. Then we can define a matrix system whose resolution is equivalent to minimise the cost function of the optimal control problem. As this is an ill-posed Cauchy problem, a regularization term must be applied on the matrix system to get physiologically acceptable solution. In this paper, we first introduce principles of the factorization by deriving equations in the simpler 3D case of a cylinder. Then, we present equations derived in the most $3 \mathrm{D}$ generalized case. This is applied to a human torso geometry case where numerical simulations are performed by using synthetical data based on the ECG solver [8]. We analyse the accuracy of the method by comparing reconstructed and synthetical solutions with RE and $\mathrm{CC}$ computations and illustrations of reconstructed potential fields.

\section{METHODS}

In this section we present the mathematical formulation of the factorization method. For the sake of clarity we first derive the equation in the 3D cylindrical case. Then, we present the mathematical formulation of the factorization method in the 3D general case.

\subsection{Initial problem}

For a given BSPs data $T$, the electrical potential $u$ in the torso domain $\Omega$ satisfies the following system $\left(\mathcal{P}_{0}\right)$ :

$$
\begin{cases}\Delta u=0 \text { in } \Omega, & \Omega: \text { cylinder } \\ u=0 \text { on } \Sigma, & \Sigma: \text { lateral surface } \\ u=T \text { on } \Gamma_{T}, & \Gamma_{T}: \text { torso surface } \\ \nabla u \cdot \mathbf{n}=0 \text { on } \Gamma_{T}, \mathbf{n}: \text { outward normal to } \Gamma_{T}\end{cases}
$$

The aim of solving the inverse problem is to find the trace of $u$ on the on the heart surface $\Gamma_{H}$. 


\subsection{Optimal control problem}

Following [7], we decompose $\left(\mathcal{P}_{0}\right)$ into two auxiliary subproblems :

$$
\left\{\begin{array} { l } 
{ \Delta u ^ { 1 } = 0 \text { in } \Omega } \\
{ u ^ { 1 } = 0 \text { on } \Sigma } \\
{ u ^ { 1 } = T \text { on } \Gamma _ { T } } \\
{ \nabla u ^ { 1 } \cdot n = \eta \text { on } \Gamma _ { H } }
\end{array} \text { and } \quad \left\{\begin{array}{l}
\Delta u^{2}=0 \text { in } \Omega \\
u^{2}=0 \text { on } \Sigma \\
u^{2}=\tau \text { on } \Gamma_{H} \\
\nabla u^{2} \cdot n=0 \text { on } \Gamma_{T}
\end{array}\right.\right.
$$

We then define the cost function as follows :

$$
E(\eta, \tau)=\int_{\Omega}\left(\nabla u^{1}(\eta)-\nabla u^{2}(\tau)\right)^{2}
$$

When $E$ reaches zero, we have $u^{1}=u^{2}$, and both of them satisfy $\left(\mathcal{P}_{0}\right)$. Our goal is then to minimise $E$. In the following paragraph, we present the factorization approach allowing to solve $\left(\mathcal{P}_{0}\right)$. This problem is ill-posed [6], we then add a regularization term to avoid the ill-posedness. The cost function becomes :

$$
E(\eta, \tau)=\int_{\Omega}\left(\nabla u^{1}(\eta)-\nabla u^{2}(\tau)\right)^{2}+\epsilon \int_{\Gamma_{H}}\left(\eta^{2}+\tau^{2}\right)
$$

where $\epsilon$ is the regularization parameter.

\subsection{Principle of invariant embbeding}

The main idea of this approach is to "transport" potential data from torso surface to the heart surface as illustrated in Figure 1. For this, boundary value problems (2) are embedded into a family of similar problems on subdomains $\Omega_{s}$. They are bounded by a moving boundary $\Gamma_{s}$ defined at $x=s$ for $x=$ $0 \longrightarrow x=a$, where $a$ is the length of the cylinder, $x=0$ corresponding to the torso surface, $x=a$ corresponding to the heart surface and $s$ is the variable that describes the axis of evolution. At each position $x=s$, we impose a Neumann boundary condition $\frac{\partial u_{s}^{1}}{\partial x}{ }_{\mid \Gamma_{s}}=\alpha$ for the $u^{1}$ problem and a Dirichlet boundary condition $\left(u_{s}^{2}\right)_{\mid \Gamma_{s}}=\beta$ for the $u^{2}$ problem

$$
\left\{\begin{array} { l } 
{ \Delta u _ { s } ^ { 1 } = 0 \text { in } \Omega _ { s } } \\
{ u _ { s } ^ { 1 } = 0 \text { on } \Sigma _ { s } } \\
{ u _ { s } ^ { 1 } = T \text { on } \Gamma _ { T } } \\
{ \nabla u _ { s } ^ { 1 } \cdot n = \alpha \text { on } \Gamma _ { s } }
\end{array} \quad \text { (5) } \left\{\begin{array}{l}
\Delta u_{s}^{2}=0 \text { in } \Omega_{s} \\
u_{s}^{2}=0 \text { on } \Sigma_{s} \\
u_{s}^{2}=\beta \text { on } \Gamma_{s} \\
\nabla u_{s}^{2} \cdot n=0 \text { on } \Gamma_{T} .
\end{array}\right.\right.
$$

At each position $x=s$, we define on $\Gamma_{s}$ an affine mapping :

Neumann to Dirichlet $\alpha \longrightarrow u_{s}^{1}: u_{s}^{1}=Q(s) \alpha+w_{1}(s)$

and a linear mapping :

$$
\text { Dirichlet to Neumann } \beta \longrightarrow \frac{\partial u_{s}^{2}}{\partial x}: \frac{\partial u_{s}^{2}}{\partial x}=P(s) \beta
$$

where $w$ is the residual associated to the operator $Q$. Folloowing [4], we substitute equations (7) in (5) and (8) in (6)

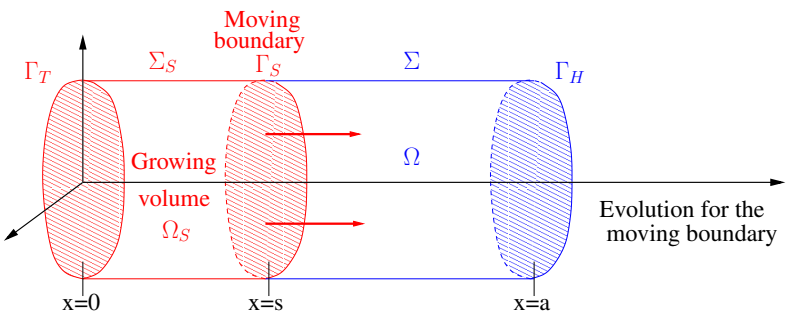

Fig. 1. Illustration of the moving boundary for the cylinder case.

to obtain the following system :

$$
\begin{cases}\frac{d P}{d x}+P^{2}=-\Delta_{y}, & P(0)=0 \\ \frac{d Q}{d x}-Q \Delta_{y} Q=I, & Q(0)=0 \\ \frac{d w_{1}}{d x}-Q \Delta_{y} w=0, & w(0)=T\end{cases}
$$

where $I$ is the identity function, $\Delta_{y}$ is the laplacian operator defined on the section $\Gamma_{s}$. Operators $P$ and $Q$ satisfy Riccati equations, whereas the residual term $w$ satisfies an ordinary differential equation depending on the operator $Q$. All the initial conditions are known and are defined on the torso surface.

According to [6], the energy function $E(\eta, \tau)$ could be rewritten using $P, Q$ and $w$ as follows :

$$
\begin{aligned}
E(\eta, \tau)=C+[\eta, \tau] A[\eta, \tau]^{T}-2 & <P(a) w(a), \tau> \\
& +\epsilon<\tau, \tau>+\epsilon<\eta, \eta>
\end{aligned}
$$

where :

$$
A=\left(\begin{array}{cc}
Q(a)+\epsilon I & -Q(a) P(a) \\
-P(a) Q(a) & P(a)+\epsilon I
\end{array}\right)
$$

and $C=\int_{\Gamma_{T}} T \partial_{n} w$ is a constant independent of $\tau$ and $\eta$. We compute the gradient of $E$ over $\tau$ and $\eta$ and find that the optimum of $E$ is reached for $\tau$ and $\eta$ satisfying :

$$
\begin{array}{r}
((1+\epsilon) I-Q(a) P(a)) \tau=w(a) \\
((1+\epsilon) I-P(a) Q(a)) \eta=P(a) w(a)
\end{array}
$$

Since we are only interested in computing the electrical potential on the heart surface we only solve the first equation of (12) using the Matlab Cholesky solver.

\subsection{Equations in the $3 D$ generalized case}

We name $\Gamma_{0}$ the torso skin, $\Gamma_{1}$ the heart skin and $\Omega$ the torso domain. We recall $T$ the electrical potential on the torso surface. For $0 \leq s \leq 1$, we define a familly surfaces $\Gamma_{s}$ included between the torso skin and the heart skin. This familly will represent the moving boundary. We also introduce $\Phi_{s}^{s^{\prime}}$, which 
is the application that maps the surface $\Gamma_{s}$ to the surface $\Gamma_{s^{\prime}}$, and $V(x)=\frac{d}{d s} \Phi_{0}^{s}(x)$ which could be seen as a "velocity" term. We decompose $V$ into a normal term $\alpha \vec{n}$ and a tangential term $V_{\Gamma_{s}}$ of the surface $\Gamma_{s}$.

Following the same derivation process from the equation (5) to the equation (9), we can establish the variational formulation of $P$ and $Q$ operators and the residuals term $w$. We use the first order finite element method to discretize theses equations on $\Gamma_{s}$. We denote $\left(e_{j}\right)_{1}^{N}$ the finite element basis allowing to approximate $P, Q$ and $w$ by $P_{h}, Q_{h}$ and $w_{h}$. Following [9] we obtain :

$$
\begin{gathered}
\frac{d}{d s}\left(M P_{h}\right)+P_{h}^{T} M^{\alpha} P_{h}+B P_{h}+\left(B P_{h}\right)^{T}=K^{\alpha} \\
P_{h}(0)=0 \\
M \frac{d Q_{h}}{d s}-\frac{d M}{d s} Q_{h}+Q_{h}^{T} K^{\alpha} Q_{h}-B^{T} Q_{h} \\
-\left(B^{T} Q_{h}\right)^{T}=M^{\alpha}, \quad Q_{h}(0)=0 \\
M \frac{d w_{h}}{d s}+Q_{h}^{T} K^{\alpha} w_{h}-B^{T} w_{h}=0, \quad w_{h}(0)=T
\end{gathered}
$$

where $M$ and $M^{\alpha}$ are mass matrices, $K^{\alpha}$ a stiffness and $B$ is the matrix defined by $B_{i, j}=<V_{\Gamma_{s}} . \nabla e_{i}, e_{j}>$. The size of all the defined matrix is $\mathrm{N} \times \mathrm{N}$ where $\mathrm{N}$ is the number of nodes on the heart surface. Then, we solve (13)-(15) using a first order explicit Euler scheme to obtain $P_{h}(1), Q_{h}(1)$ and $w_{h}(1)$. Finally we obtain the heart potential by solving the linear system (12).

\section{RESULTS}

A computational geometry of a human torso volume was produced from a 43 years old women anatomical model. We used the bidomain model to simulate one second of heart beat on the heart and body surface. We extract the electrical potential on the torso boundary and use it as an input of the inverse problem. At each time step the torso potential represents the data $T$ as in equation (15). Figure 2 shows a snapshot of the torso potential (left) and the corresponding heart potential (right).

In order to generate the sequence of surfaces morphing from
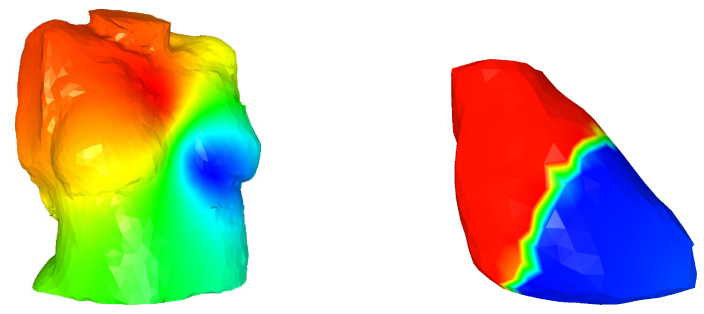

Fig. 2. Example of torso and heart potential field generated by the bidomain model. the torso surface to the heart surface, we solve the Laplace equation with a Dirichlet boundary condition equal to 1 on the torso surface and 0 on the heart surface as shown in Figure 3 (left). We then extract the surfaces as the iso-values of the solution as shown in Figure 3 (right). These iso-surfaces are
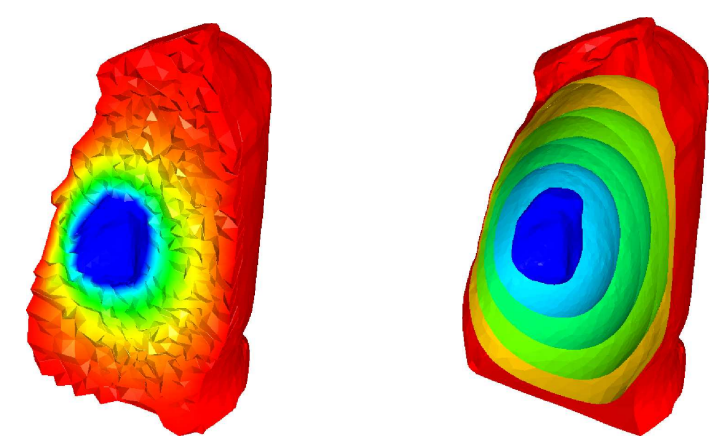

Fig. 3. Illustration of the surfaces $\Gamma_{s}$ generated. Left : isovalues computed in the torso volume. Right : surfaces created from iso-values.

used to compute the operators $P_{h}(1), Q_{h}(1)$ and $w_{h}(1)$ and construct the electrical potential on the heart surface as explained in section 2.3 for various $\epsilon$ values $\left(10^{-1}, 10^{-2}, 10^{-3}\right.$ and $\left.10^{-4}\right)$. In Figure 4, we show a comparison between the measured (left column) and the inverse solutions (right column) at times $t=100 \mathrm{~ms}$ (top) and $t=250 \mathrm{~ms}$ (bottom) for $\epsilon=10^{-4}$. We remark that time $t=100 \mathrm{~ms}$, the inverse solution is diffusive compared to the exact solution which has a sharp wave front. Whereas at time $t=250 \mathrm{~ms}$ where the exact solution is smoother and the reconstructed potential is more accurate. In Figure 5 we show the time course of the
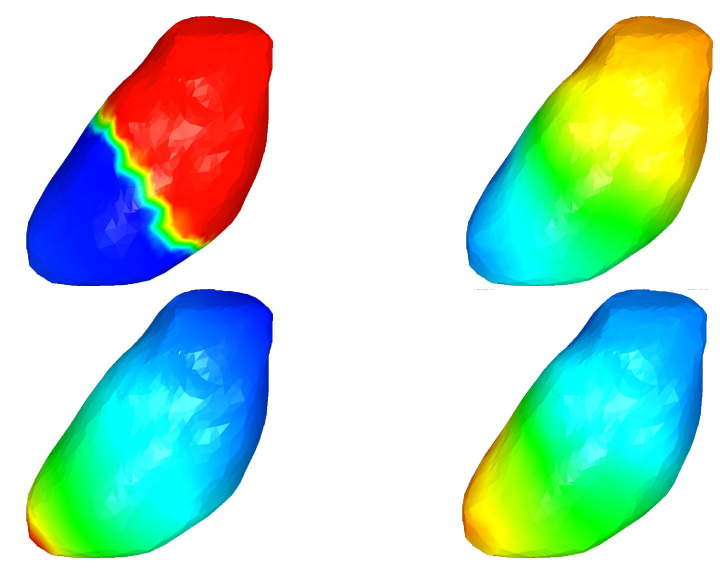

Fig. 4. Measured (left column) and reconstructed (right column) potential on the heart surface at $t=100 \mathrm{~ms}$ (top) and $250 \mathrm{~ms}$ (bottom).

relative error (RE) in space and in Figure 6 we show the time course of the correlation coefficient (CC), for the different values of the regularization parameter $\epsilon$. Although the RE is high, the average $\mathrm{CC}$ is close to 0.7 allowing to a relatively 
accurate capture of the wave front. The high RE is due to the fact that the magnitude of the reconstructed potential is smaller than the exact solution.

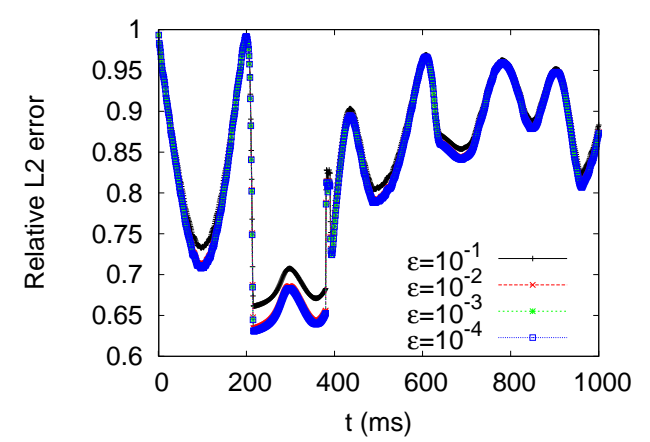

Fig. 5. Relative $L 2$ error computed for several time steps.

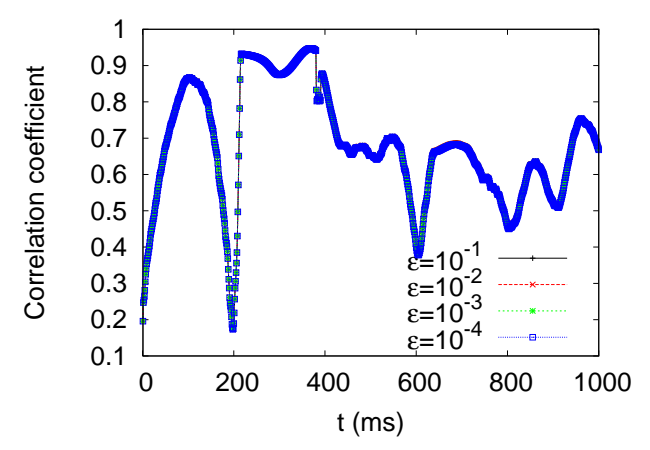

Fig. 6. Correlation coefficient computed for several time steps.

\section{DISCUSSION}

This work represents the first use of the factorization of boundary value problems method in solving the inverse problem in electrocardiography. The method was firstly introduced for general Laplace equation in 2D [6] and it was technically challenging to implement this method in a 3D framework and adapt it to the ECGI problem. The most challenging parts are : 1) generating the family of moving surfaces between the heart and the torso, 2) solving the non linear Riccati equations on the moving surfaces. We used an explicit Euler scheme to linearize and solve the Riccati equations in order to build the operators $P$ and $Q$ at the heart surface. This scheme allows to conserve the positivity and the symmetry of the operator $P$, but does not necessarily conserve the symmetry of the operator $Q$. More adapted numerical schemes should be carried out in future works in order to accurately solve the operator $Q$.

\section{CONCLUSION}

In this paper, we presented a new approach for solving the inverse problem in electrocardiography called the factoriza- tion of boundary value problems method. We derived equations of the method in a cylindrical 3D case to illustrate this approach then in the general 3D case. We have tested this method on synthetical data generated on a realistic human geometry. Since the CC is relatively high, the method could be considered as a good candidate for applications like capturing the wave front position on the heart surface. We think that RE could be increased by improving the numerical discretization of the equation solving the operator $Q$. We will also take into account the time sequences by adding a temporal regularization term into the cost function. This would be subject of future works.

\section{REFERENCES}

[1] L. A. Bokeriya, A. S. Revishvili, A. V. Kalinin, V. V. Kalinin, O. A. Lyadzhina, and E. A. Fetisova, "Hardwaresoftware system for noninvasive electrocardiographic heart examination based on inverse problem of electrocardiography," Biomedical Engineering, vol. 42, no. 6, pp. 273-279, 2008.

[2] C. Ramanathan, R. N Ghanem, P. Jia, K. Ryu, and Y. Rudy, "Noninvasive electrocardiographic imaging for cardiac electrophysiology and arrhythmia," Nature medicine, vol. 10, no. 4, pp. 422-428, 2004.

[3] Z. Liu, C. Liu, and B. He, "Noninvasive reconstruction of three-dimensional ventricular activation sequence from the inverse solution of distributed equivalent current density," Medical Imaging, IEEE Transactions on, vol. 25, no. 10, pp. 1307-1318, 2006.

[4] J. Henry and A. M. Ramos, "Factorization of secondorder elliptic boundary value problems by dynamic programming," Nonlinear Analysis: Theory, Methods \& Applications, vol. 59, no. 5, pp. 629-647, 2004.

[5] F. Jday, "Complétion de données frontières : la méthode de plongement invariant," PHD thesis, 2013.

[6] A. Ben Abda, J. Henry, and F. Jday, "Boundary data completion: the method of boundary value problem factorization," Inverse Problems, vol. 27, no. 5, pp. 055014, 2011.

[7] S. Andrieux, T. N. Baranger, and A. B. Abda, "Solving cauchy problems by minimizing an energy-like functional," Inverse problems, vol. 22, no. 1, pp. 115, 2006.

[8] N. Zemzemi, Étude théorique et numérique de lactivité électrique du cour: Applications aux électrocardiogrammes, Ph.D. thesis, Paris 11, 2009.

[9] J. Bouyssier, N. Zemzemi, and J. Henry, “3d generalisation of the factorization of boudary values problem method : application to the electrocardiography inverse problem," Tech. Rep., INRIA, 2015. 